\title{
Multiscale patterning of graphene oxide and reduced graphene oxide for flexible supercapacitors
}

\author{
Yuhua Xue $e^{\mathrm{a}, \mathrm{b}}$, Lin $\mathrm{Zhu}^{\mathrm{a}, \mathrm{b}}$, Hao Chen ${ }^{\mathrm{a}, *}, \mathrm{Jia}^{\mathrm{Q}} \mathrm{u}^{\mathrm{a}, *}$, Liming Dai ${ }^{\mathrm{a}, \mathrm{b}, *}$ \\ ${ }^{a}$ Institute of Advanced Materials for Nano-Bio Applications, School of Ophthalmology \\ \& Optometry, Wenzhou Medical College, 270 Xueyuan Xi Road, Wenzhou, Zhejiang \\ 325027, China \\ ${ }^{\mathrm{b}}$ Center of Advanced Science and Engineering for Carbon (Case4Carbon), \\ Department of Macromolecular Science and Engineering, \\ Case Western Reserve University, 10900 Euclid Avenue, Cleveland, Ohio 44106, USA
}

\begin{abstract}
A simple and facile method for multiscale, in-plane patterning of graphene oxide and reduced graphene oxide (GO-rGO) was developed by region-specific reduction of graphene oxide (GO) under a mild irradiation. The UV-induced reduction of graphene oxide was monitored by various spectroscopic techniques, including optical absorption, X-ray photoelectron spectroscopy (XPS), Raman, and X-ray diffraction (XRD), while the resultant GO-rGO patterned film morphology was studied on optical microscope, scanning electron microscope (SEM), and atomic force microscope (AFM). Flexible symmetric and in-plane supercapacitors were fabricated from the GO-rGO patterned polyethylene terephthalate (PET) electrodes to show capacitances up to $141.2 \mathrm{~F} / \mathrm{g}$.
\end{abstract}

\footnotetext{
*Corresponding authors: Tel: +1 216 4176. E-mail address: chenhao@mail.eye.ac.cn (Hao Chen); jia.qu@163.com (Jia Qu); liming.dai@ case.edu (Liming Dai)
} 


\section{Introduction}

Graphene, an atomic thick sheet of two dimensional $\mathrm{sp}^{2}$ carbon structure, has received considerable attention due to its unique structure and extraordinary properties, such as large specific surface area, good optoelectronic characteristics, and high mechanical strength [1-3]. Thus, graphene holds great potentials for various applications in devices for energy conversion and storage [4, 5], including supercapacitors [6], solar cells [7], batteries [8], and fuel cells [9]. Various methods, such as chemical vapor deposition (CVD), mechanical exfoliation, ball milling, and reduction of graphene oxide (GO) into reduced graphene oxide (rGO), have been devised for graphene preparation [1, 4-6]. Among them, the chemical exfoliation of graphite to produce graphene oxide, followed by reduction, remains the most promising method to obtain graphene materials because of its low cost, high yield, and good solution processability [1].

For certain device applications, large-scale patterning of graphene is highly desirable $[10,11]$. It is still a big challenge to pattern graphene [11], though some rGO patterns have been obtained by lithographically patterning/reducing GO films $[11,12]$. However, the lithographic patterning involves multiple procedures and is time-consuming. Inkjet printing $[13,14]$ and layer-by-layer assembling [15] have also been developed for micropatterning $\mathrm{GO}$ for device applications. Of particular interest, direct laser writing/reducing of GO films [16-19] has been demonstrated to be a powerful approach to micropatterns with $\mathrm{rGO}$ regions interposed into the GO matrix without the needs to etch the undesirable GO parts, attractive for fabricating all carbon devices. Nevertheless, the strong laser used often causes structure damages to the graphene oxide film while the laser equipment is expensive and not easy to operate. To develop simple and facile 
method for large-scale patterning of rGO films without structural damage, UV light can be used as alternative optical source to make large-scale GO-rGO patterns. Indeed, UV light has been used to reduce GO in aqueous solutions [20, 21]. Compared with a laser beam, UV light is much milder and much easier to operate.

In this study, a series of multiscale ( $\mu \mathrm{m}$ to $\mathrm{cm}$ ) GO-rGO patterns were prepared via region-specific UV-induced mild reduction of GO films. The resultant large-scale GO-rGO patterns were used to construct flexible two-electrode, solid-state symmetric supercapacitors and in-plane supercapacitors with good performance.

\section{Experimental}

\subsection{Preparation of GO and UV induced GO-rGO patterns:}

GO was synthesized via a modified Hummers method according to procedures published elsewhere [22]. To prepare the GO-rGO patterns, $3 \mathrm{ml}$ of $0.4 \mathrm{~mol} / \mathrm{L} \mathrm{GO}$ solution was filtrated through a PVDF filter membrane with a pore size of $0.22 \mu \mathrm{m}$. The resulting GO film on PVDF filter membrane was transferred to a transparent polyethylene terephthalate (PET) substrate, and then peeled off the PVDF membrane by spray some acetone on the back side of the PVDF film. The GO film on PET substrate was then covered by a quartz photomask with a desired pattern structure. The photomasked sample was then put under a UV lamp (Blak-Ray longwave Ultraviolet Lamp, Model B-100AP, $115 \mathrm{~V}, 60 \mathrm{~Hz}, 2.5 \mathrm{~A}$ ) for irradiation for various irradiation durations from 1 hour to 24 hours. Finally, the GO-rGO pattern was obtained upon removing the photomask. Transparent plastic (e.g., PET) films with pre-printed patterns (e.g., university logos) can also be used as photomasks. 
2.2. Fabrication of flexible solid-state symmetric supercapacitors.

Preparation of the UV reduced graphene oxide (UV-rGO) electrode: A thin layer of Au was first sputter-coated on a PET film, and then the Au-PET film was cut into $1 \mathrm{~cm}$ $\times 2 \mathrm{~cm}$ slides as the current collector. Thereafter, $1 \mathrm{ml}$ of GO solution $(0.4 \mathrm{~mol} / \mathrm{L})$ was drop coated on the Au-PET slide and dried overnight at room temperature $\left(20^{\circ} \mathrm{C}\right)$. The resultant GO film on Au-PET slide was exposed to the UV irradiation for 24 hours to produce the UV-rGO electrode.

Fabrication of the symmetric supercapacitors: The $\mathrm{H}_{3} \mathrm{PO}_{4} / \mathrm{PVA}$ gel electrolyte was prepared by mixing an aqueous solution of PVA $\left(1 \mathrm{~g}\right.$ PVA in $\left.10 \mathrm{~mL} \mathrm{H}_{2} \mathrm{O}\right)$ with concentrated $\mathrm{H}_{3} \mathrm{PO}_{4}(0.8 \mathrm{~g})$, which was then spin-coated on the UV-rGO electrode and kept in fume hood to vaporize water for two hours. Then, two such UV-rGO electrodes were pressed together with a $\mathrm{H}_{3} \mathrm{PO}_{4} / \mathrm{PVA}$ electrolyte layer being sandwiched between them to make the all-solid-state symmetric supercapacitor.

\subsection{Fabrication of flexible solid-state in-plane supercapacitors.}

The PET-supported GO film was exposed to the UV irradiation through a photomask with an interdigited pattern for 24 hours. After removal of the photomask, the GO-rGO patterns were evident. The $\mathrm{H}_{3} \mathrm{PO}_{4} / \mathrm{PVA}$ gel electrolyte was then spin-coated on the in-plane patterned GO-rGO film and dried at room temperature. Two edge-sides of the GO-rGO patterned film was sputter-coated with Au as the current collectors.

\subsection{Characterization.}

X-ray photoelectron spectroscopic (XPS) measurements were carried out on a PHI 5000 VersaProbe. Raman spectra were collected using a Raman spectrometer 
(Renishaw) with a $514 \mathrm{~nm}$ laser. X-ray diffraction (XRD) was measured on a Miniflex II Desktop X-ray diffractometer. UV-vis transmittance was measured on a Jasco V-670 spectrophotometer. Atomic force microscopic imaging was done on an Agilent Technologies 5500 Scanning Probe Microscope. Scanning electron microscopic (SEM) images were taken on JEOL JSM-6510LV SEM. The Cyclic voltammograms (CVs) were measured on a computer-controlled potentiostat (CHI 760C, CH Instrument, USA).

\section{Results and discussion:}

Figure 1A schematically shows the route to GO-rGO patterns. To start with, a GO film was solution coated on a substrate, which can be either soft (e.g., a PET film) or hard one (e.g., Si, glass plates). Prior to exposure to the UV light, a photo mask with a desired pattern structure was placed on the GO film. The graphene oxide within the exposed regions was then reduced into rGO under the UV irradiation [16, 22]. Finally, the GOrGO pattern was obtained after the removal of the photo mask.

As shown in Figure 1B, we have prepared a series of GO-rGO patterns with various geometrical structures and different resolutions. These rGO-GO patterns can be easily identified even on an optical microscope due to the big contrast in optical transparency between the rGO and GO areas; the UV-reduced graphene oxide regions became much darker than the GO region. Uniformly micropatterned thin rGO lines with a resolution up to about $10 \mu \mathrm{m}$ were evident in Figure $1 \mathrm{~B}(\mathrm{e})$, while laser-jet printed patterns on transparent plastic (e.g., PET) films could be used as photo masks, as exemplified by Figures 1B(c\&f). 


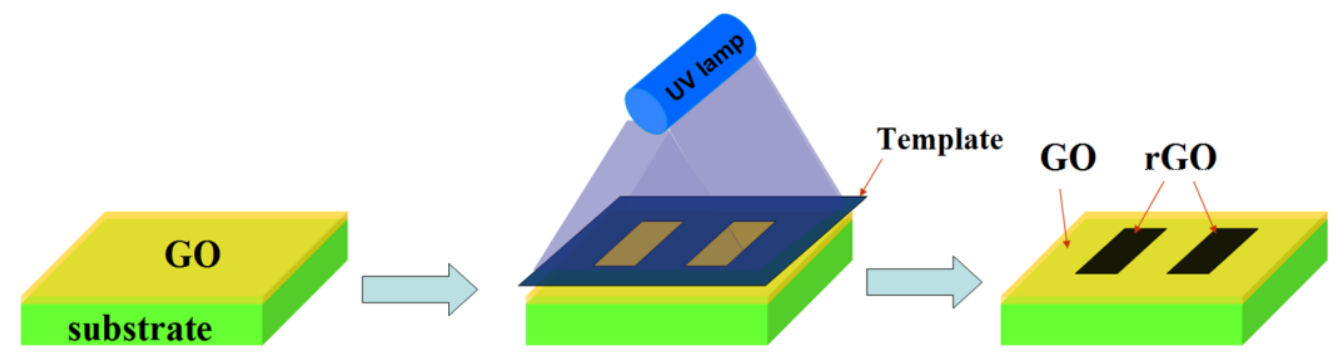

(A)

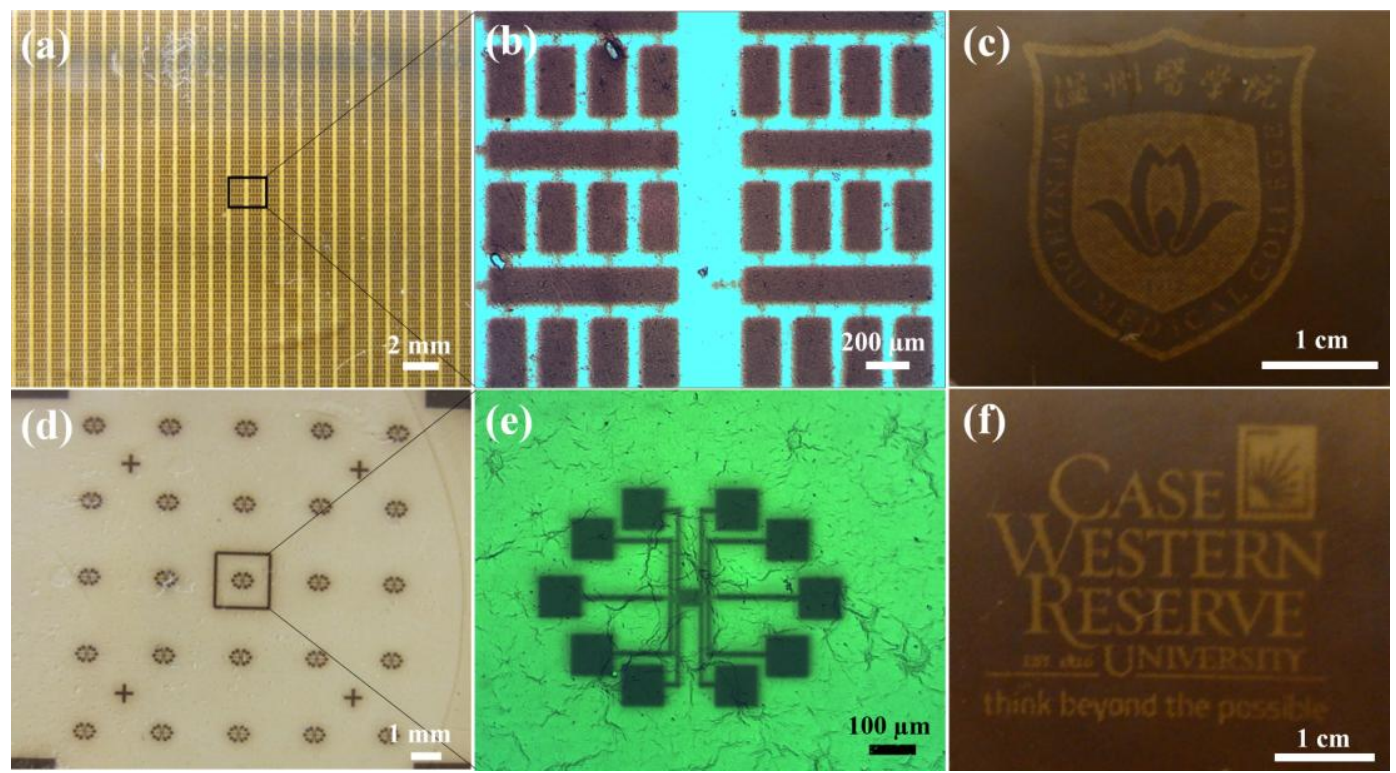

(B)

Figure 1. (A) Schematic representation of the route to the GO-rGO pattern. (B) Photographs of GO-rGO patterns: (a) GO-rGO pattern with aligned rGO ribbons. (b) An enlarged view of the black box in image (a). (c) A logo of Wenzhou Medical University made from the GO-rGO pattern. (d) A series patterns of GO-rGO. (e) An enlarged view of the black box in image (d). (f) A logo of Case Western Reserve University made from the GO-rGO pattern. PET films with pre-printed respective university logos were used as photomasks for (c \& f). 
The use of PED films as the support for GO coating allows for the preparation of transparent and flexible GO-rGO patterned films for potential flexible device applications, as demonstrated in Figures 2a\&b.
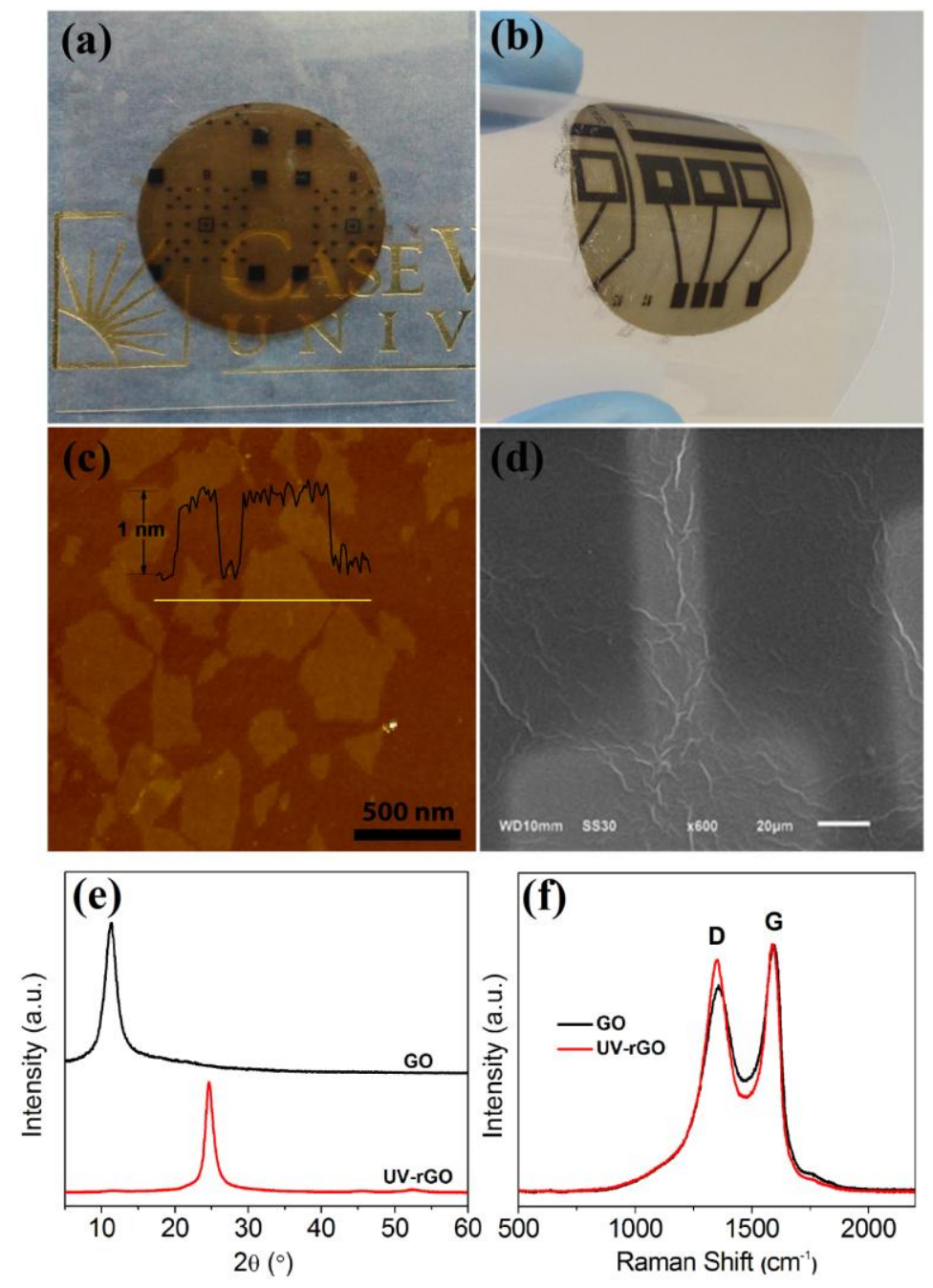

Figure 2. $(\mathrm{a}, \mathrm{b})$ photographs of GO-rGO patterns on the transparent and flexible PET substrates. (c) An AFM image of GO, (d) A SEM image of the GO-rGO pattern, (e) XRD of GO and UV-rGO films. (f) Raman spectra of GO and UV-rGO.

To investigate the microstructure and morphology of these GO-rGO micropatterns, we performed AFM and SEM imaging. As seen in Figure 2c, the 
thickness of GO is about $1 \mathrm{~nm}$. In Figure 2d, the white area corresponds to rGO while the grey area represents GO with uniform morphologies in respective regions and an intimate interface. The reduction of GO film by the UV irradiation is confirmed by X-ray diffraction (XRD) and Raman spectroscopic measurements carried out with a nonpatterned GO film before and after the UV irradiation under the same condition without using a photo mask. As shown in Figure 2e, the GO film shows a diffraction profile with a peak at $2 \theta=11.2^{\circ}$ corresponding to an interlayer distance of $0.79 \mathrm{~nm}$ in consistent with reported data $[23,24]$. The UV-reduced rGO shows a broad XRD peak at about $2 \theta=25^{\circ}$ corresponding to the interlayer distance of about $0.40 \mathrm{~nm}$, which is in a good agreement with the graphene interlayer distance [23]. These results indicate the occurrence of GO reduction by the UV irradiation and $\pi-\pi$ stacking of the reduced graphene oxide. The reduction of GO film by the UV irradiation was also confirmed by Raman spectra given in Figure $2 \mathrm{f}$, which shows the $I_{\mathrm{D}} / I_{\mathrm{G}}$ peak intensity ratios of about 0.837 and 0.937 for GO and $\mathrm{rGO}$, respectively, indicating a reduction in the average size of $s p^{2}$ domains with edge defects caused by the reduction of some $\mathrm{C}=\mathrm{C}$ bonds in $\mathrm{GO}$ [24].

The kinetic process of the UV irradiation reduction of GO was monitored by the optical transmittance and XPS measurements at different UV-irradiation times from 1 to 24 hours. As can be seen in Figures 3A, the samples were put on a paper with parallel lines as the background, the GO films became darker with increasing the irradiation time. The corresponding transmittance spectra over the visible wavelengths are shown in Figure 3B, which also shows that the GO film become less transparent after UV irradiation, and that transmittance over the entire visible region decreased with increasing the UV-irradiation time. After UV irradiation, the resulted UV-reduced graphene oxide 
also became electrically conducting with a resistance of $47 \mathrm{~K} \Omega / \mathrm{cm}$ for the $\mathrm{rGO}$ film obtained by UV irradiation for 24 hours.
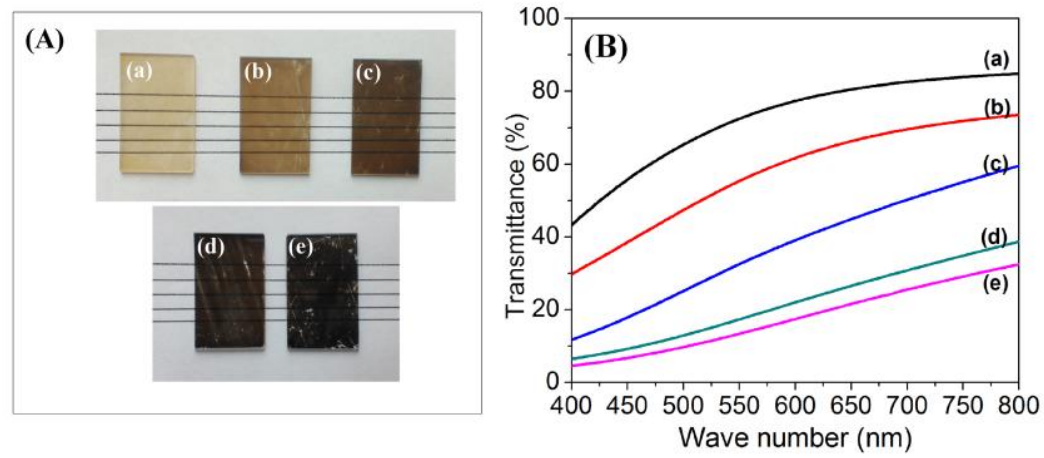

Figure 3. (A) Photographs and (B) transmittance curves of the GO film (a) before and after the UV irradiation for different durations: (b) 1 hour, (c) 6 hours, (d) 12 hours, and (e) 24 hours.

Figure 4 shows XPS results of GO before and after the UV irradiation. As shown in Fiugre 4A and Table 1, C1s (65 at\%) and O1s (35 at\%) peaks were seen in the XPS survey spectrum for GO before the UV reduction (Figure 4A(a)). Upon the UV irradiation, the oxygen atomic content decreased from $35 \%$ for GO to $20 \%$ in the UVrGO after the UV exposure for 24 hours (Figure 4A(e)). The corresponding C/O ratio increased from 1.9 to 5, indicating, once again, the partial reduction of GO. The highresolution C1s XPS spectra for GO and UV-rGO at different UV irradiation durations are shown in Figure 4B. As can be seen, the C1s peak can be fitted mainly with four components at $284.5 \mathrm{eV}(\mathrm{C}=\mathrm{C}), 286.5 \mathrm{eV}(\mathrm{C}-\mathrm{O}), 288.1 \mathrm{eV}(\mathrm{C}=\mathrm{O})$ and $289.0 \mathrm{eV}(\mathrm{COOH})$, respectively. The UV irradiation caused a graduate decrease in the C-O peak intensity at $286.5 \mathrm{eV}$ whereas the peak intensities of $\mathrm{C}=\mathrm{O}(288.1 \mathrm{eV})$ and $\mathrm{COOH}(289.0 \mathrm{eV})$ 
remained largely unchanged, indicating that UV irradiation reduced mainly the $\mathrm{C}-\mathrm{O}$ groups.
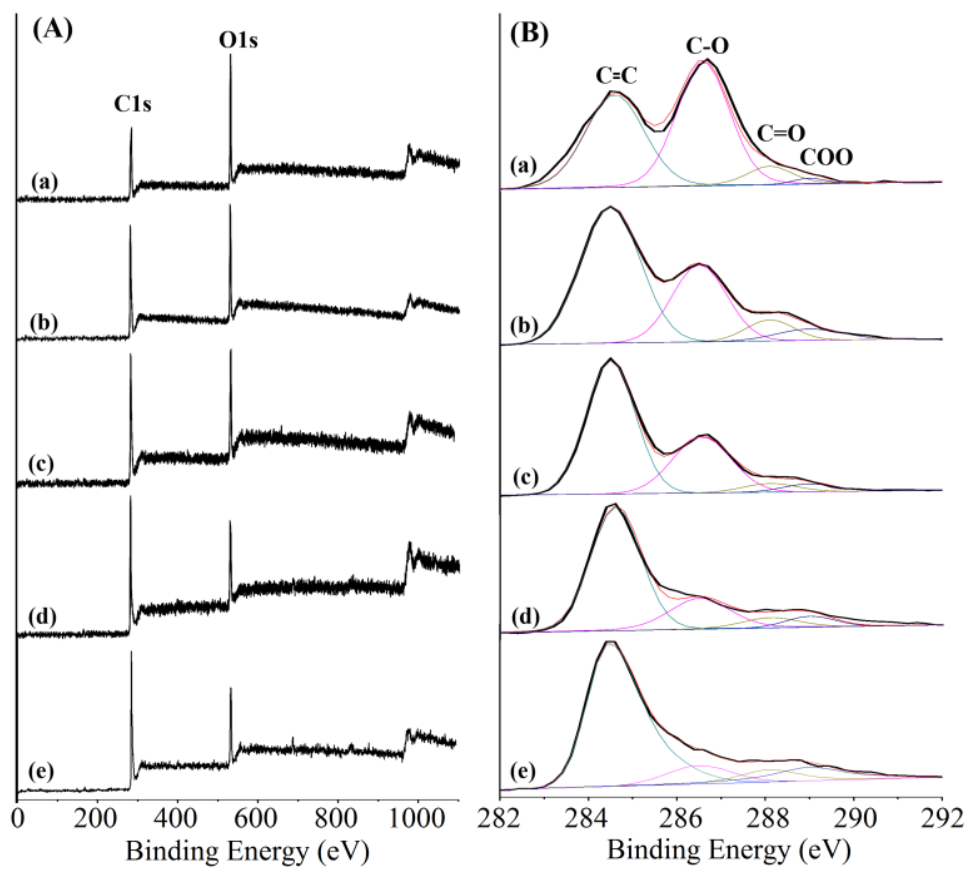

Figure 4. (A) The survey and (B) high resolution C1s XPS spectra of (a) GO and UVrGO at different UV-irradiation times: (b) 1 hour, (c) 6 hours, (d) 12 hours, and (e) 24 hours.

Table 1. Element contents of the UV reduced graphene with different UV irradiation times.

\begin{tabular}{ccc}
\hline $\begin{array}{c}\text { UV irradiation times } \\
\text { (hours) }\end{array}$ & C (atom\%) & O (atom\%) \\
\hline $\mathbf{0}$ & 65.0 & 35.0 \\
\hline $\mathbf{1}$ & 72.1 & 27.9 \\
\hline $\mathbf{6}$ & 75.1 & 24.9 \\
\hline $\mathbf{1 2}$ & 77.9 & 22.1 \\
\hline $\mathbf{2 4}$ & 80.0 & 20.0 \\
\hline
\end{tabular}

To demonstrate potential applications of the UV-reduced rGO in supercapacitors, we constructed traditional solid-state symmetric supercapacitors by sandwiching the 
$\mathrm{H}_{3} \mathrm{PO}_{4} / \mathrm{PVA}$ gel electrolyte between two 24-hour UV-rGO electrodes (Figure 5a, Section 2.2). CV curves of the UV-rGO supercapacitor are given in Figure 5b, which shows a very regular rectangle shape characteristic of excellent double layer charge storage. The rectangular $\mathrm{CV}$ curve persisted even at the high scan rate of $1 \mathrm{~V} / \mathrm{s}$, indicating a rapid charging and discharging process with a low series resistance [25]. The charge and discharge $(\mathrm{CD})$ curves are presented in Figure 5c, from which the specific capacitances were calculated using the following equation [26, 27],

$$
C s=4 \times \frac{I \Delta t}{U m}
$$

where $I$ is the applied current, $\Delta t$ is the discharge time, $m$ is the total mass of the UV-rGO on both electrodes, and $U$ is the potential range. It was found that the capacitance of the UV-rGO capacitor is $141.2 \mathrm{~F} / \mathrm{g}$ at the charge and discharge current density of $1 \mathrm{~A} / \mathrm{g}$. Figure $5 \mathrm{~d}$ shows the capacitance as a function of the current density, revealing that the specific capacitance initially decreased with increasing the current density, and then leveled off above $c a .5 \mathrm{~A} / \mathrm{g}$. Our capacitor also showed a high stability over 2000 cycles (Figure 5e). 


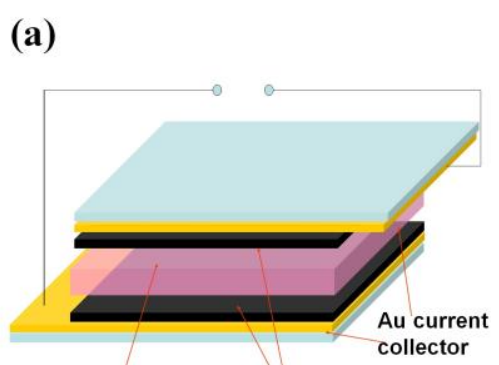

Polymer electrolyte UV-rGO
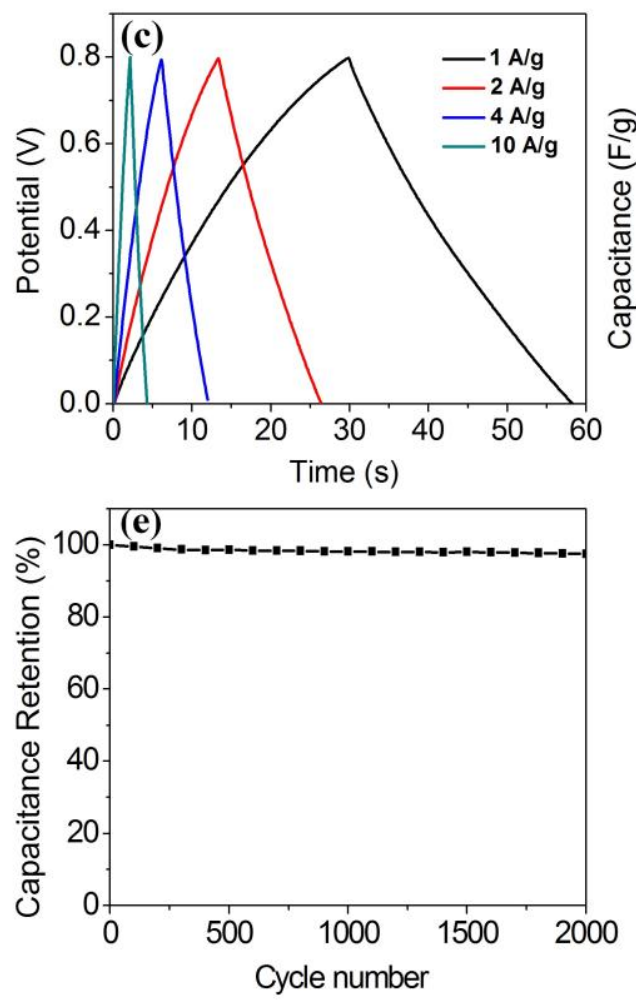
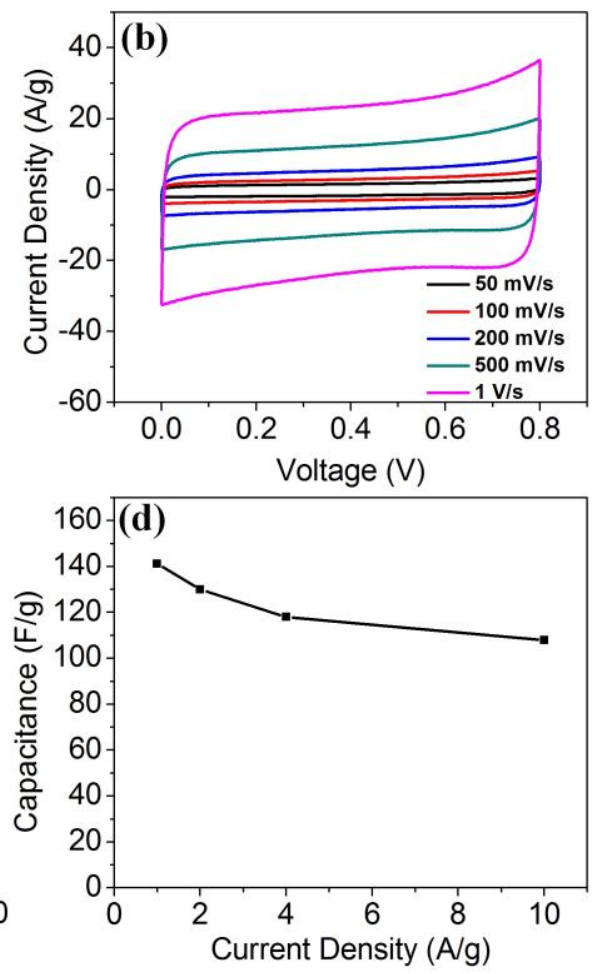
We have further constructed in-plane supercapacitors by UV-induced patterning of GO-rGO with the rGO patterns interposed in the GO matrix. Figure 6a shows a photograph of the in-plane supercapacitor based on the interdigited GO-rGO electrodes. A CV curve for the in-plane supercapacitor is shown in Figure 6b, which demonstrates that the in-plane device concept works. Although the performance of the in-plane GOrGO supercapacitor is still far from ideal, there is considerable room for further improvement of its performance by optimizing the device structure and materials quality.
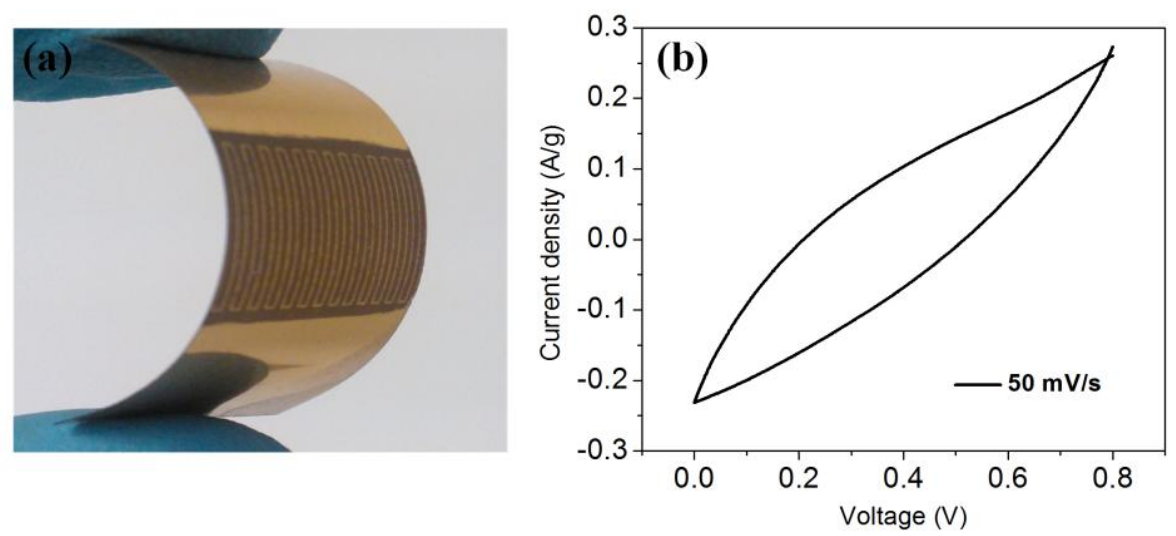

Figure 6. (a) Photograph of a flexible in-plane supercapacitor based on the in-plane patterned GO-rGO interdigitated electrodes. (b) A CV curve of the flexible in-plane supercapacitor.

\section{Conclusions}

We have developed a facile method for large-scale, in-plane patterning of GOrGO via a mild UV-induced reduction of GO by region-specific UV exposure through a photomask. Various multi-scale GO-rGO patterns with different resolutions have been prepared. The UV-induced GO reduction was confirmed by XPS and Raman spectroscopic measurements. Optical microscopic and SEM imaging show that the UV 
reduction of GO did not cause any damage on the macroscopic morphology of the GO film. Using these patterned UV-rGO electrodes, we have also fabricated flexible symmetric and in-plane supercapacitors with capacitances up to $141.2 \mathrm{~F} / \mathrm{g}$.

\section{Acknowledgments}

This work was supported financially by AFOSR (FA-9550-12-1-0069), DOD-Army (W911NF-11-1-0209), NSF (CMMI-1400274, IIP-1343270), NSFC-NSF (DMR1106160), CWRU-WMU (CON115346), NSFC (51202167), and the "Thousand Talents Program" of China.

\section{REFERENCES}

[1] Allen MJ, Tung VC, Kaner RB. Honeycomb carbon: a review of graphene. Chem Rev 2010;110:132-45.

[2] Novoselov KS, Geim AK, Morozov SV, Jiang D, Zhang Y, Dubonos SV, et al. Electric field effect in atomically thin carbon films. Science 2004;306:666-9.

[3] Tsen AW, Brown L, Levendorf MP, Ghahari F, Huang PY, Havener RW, et al. Tailoring electrical transport across grain boundaries in polycrystalline graphene. Science 2012;336:1143-6.

[4] Dai LM. Functionalization of graphene for efficient energy conversion and storage. Accounts Chem Res 2013;46:31-42.

[5] Liu J, Xue YH, Zhang M, Dai LM. Graphene-based materials for energy applications. MRS Bull 2012;37:1265-72. 
[6] Huang Y, Liang JJ, Chen YS. An overview of the applications of graphene-based materials in supercapacitors. Small 2012;8:1805-34.

[7] Xue YH, Liu J, Chen H, Wang RG, Li DQ, Qu J, et al. Nitrogen-doped graphene foams as metal-free counter electrodes in high-performance dye-sensitized solar cells. Angew Chem Int Edit 2012;51:12124-7.

[8] Yoo E, Kim J, Hosono E, Zhou H, Kudo T, Honma I. Large reversible Li storage of graphene nanosheet families for use in rechargeable lithium ion batteries. Nano Lett 2008;8:2277-82.

[9] Xue YH, Yu DS, Dai LM, Wang RG, Li DQ, Roy A, et al. Three-dimensional B,Ndoped graphene foam as a metal-free catalyst for oxygen reduction reaction. Phys Chem Chem Phys 2013;15:12220-6.

[10] Hong JY, Jang J. Micropatterning of graphene sheets: recent advances in techniques and applications. J Mater Chem 2012;22:8179-91.

[11] Zhou Y, Loh KP. Making patterns on graphene. Adv Mater 2010;22:3615-20.

[12] Kim NH, Kim BJ, Ko Y, Cho JH, Chang ST. Surface energy engineered, highresolution micropatterning of solution-processed reduced graphene oxide thin films. Adv Mater 2013;25:894-8.

[13] Zhang L, Liu HT, Zhao Y, Sun XN, Wen YG, Guo YL, et al. Inkjet printing highresolution, large-area graphene patterns by coffee-ring lithography. Adv Mater 2012;24:436-40.

[14] Shin KY, Hong JY, Jang J. Micropatterning of graphene sheets by inkjet printing and its wideband dipole-antenna application. Adv Mater 2011;23:2113-8. 
[15] Dimiev A, Kosynkin DV, Sinitskii A, Slesarev A, Sun ZZ, Tour JM. Layer-by-layer removal of graphene for device patterning. Science 2011;331:1168-72.

[16] El-Kady MF, Strong V, Dubin S, Kaner RB. Laser scribing of high-performance and flexible graphene-based electrochemical capacitors. Science 2012;335:1326-30.

[17] Zhang YL, Guo L, Wei S, He YY, Xia H, Chen QD, et al. Direct imprinting of microcircuits on graphene oxides film by femtosecond laser reduction. Nano Today 2010;5:15-20.

[18] Wei ZQ, Wang DB, Kim S, Kim SY, Hu YK, Yakes MK, et al. Nanoscale tunable reduction of graphene oxide for graphene electronics. Science 2010;328:1373-6.

[19] Park JB, Yoo JH, Grigoropoulos CP. Multi-scale graphene patterns on arbitrary substrates via laser-assisted transfer-printing process. Appl Phys Lett 2012;101:043110. [20] Ding YH, Zhang P, Zhuo Q, Ren HM, Yang ZM, Jiang Y. A green approach to the synthesis of reduced graphene oxide nanosheets under UV irradiation. Nanotechnology $2011 ; 22$.

[21] Guardia L, Villar-Rodil S, Paredes JI, Rozada R, Martinez-Alonso A, Tascon JMD. UV light exposure of aqueous graphene oxide suspensions to promote their direct reduction, formation of graphene-metal nanoparticle hybrids and dye degradation. Carbon 2012;50:1014-24.

[22] Xue YH, Chen H, Yu DS, Wang SY, Yardeni M, Dai QB, et al. Oxidizing metal ions with graphene oxide: the in situ formation of magnetic nanoparticles on self-reduced graphene sheets for multifunctional applications. Chem Commun 2011;47:11689-91.

[23] Moon, IK, Lee, J, Ruoff, RS, Lee, H. Reduced graphene oxide by chemical graphitization. Nat Commun 2010;1:73. 
[24] Stankovich S, Dikin DA, Piner RD, Kohlhaas KA, Kleinhammes A, Jia Y, et al. Synthesis of graphene-based nanosheets via chemical reduction of exfoliated graphite oxide. Carbon 2007;45:1558-65.

[25] Yu DS, Dai LM. Self-assembled graphene/carbon nanotube hybrid films for supercapacitors. J Phys Chem Lett 2010;1:467-70.

[26] Xie K, Qin XT, Wang XZ, Wang YN, Tao HS, Wu Q, et al. Carbon nanocages as supercapacitor electrode materials. Adv Mater 2012;24:347-52.

[27] Simon P, Gogotsi Y. Materials for electrochemical capacitors. Nat Mater 2008;7:845-54. 